\title{
Die Alterssicherung des öffentlichen Dienstes in den Nordischen Ländern Dänemark, Norwegen und Schweden
}

Peter A. Köhler

I. Dänemark

1. Alterssicherung nach dem allgemeinen System

2. Alterssicherung der „Tjenestemænd“ (Beamte)

a) Das „Beamtenstatut“"

b) Die Pensionsordnungen

3. Reformen

II. Norwegen

1. Alterssicherung nach dem allgemeinen System

a) Das bisher geltende Recht

b) Das ab dem 1. Januar 2010 geltende Recht

c) Finanzierung

2. Alterssicherung der „Tjenestemenn“ (Beamte)

a) Das ,Beamtenstatut"“

b) Die Pensionsordnungen

3. Reformen

III. Schweden

1. Alterssicherung nach dem allgemeinen System

2. Alterssicherung der „Tjenestemenn“ (Beamte)

a) Das „Beamtenstatut"

b) Die Pensionsordnung

3. Reformen

IV. Bewertung 
In der internationalen sozialpolitischen Diskussion ist allgemein meist von den „Nordischen Wohlfahrtsstaaten“ oder von einem „Nordischen Modell der sozialen Sicherheit" die Rede. Bei einem genaueren Blick auf die einzelnen Länder zeigt sich jedoch schnell: „The concept ,Norden' is regarded as a model with five exceptions."1

Als Gemeinsamkeit dieser Länder galt lange Zeit, daß ihre Systeme der sozialen Sicherheit primär steuerfinanziert und „universalistisch“ in dem Sinne waren, als der Kreis der Versicherten durch den Wohnsitz im jeweiligen Land bestimmt wurde. Dies gilt zumindest hinsichtlich der Alterssicherung heute nicht mehr ausnahmslos. Hier sollen die Alterssicherungssysteme Dänemarks, Norwegens und Schwedens in den Rechtsvergleich einbezogen werden, um womöglich für den öffentlichen Dienst geltende Besonderheiten zu erkennen.

\section{Dänemark}

Dänemark gehört zu den Ländern Europas, die bereits im 19. Jahrhundert begonnen haben, die Sicherung des Lebensunterhalts alter Menschen als Staatsaufgabe zu erkennen. Mit dem „Gesetz über die Altersunterstützung an würdige Bedürftige außerhalb des Armenwesens" von 1891,2 das noch dem Prinzip der Sicherung des bloßen Existenzbedarfs folgte, begann die dänische Sozialgesetzgebung. Durch das 1892 folgende Krankenkassengesetz, das den Beginn einer Volksversicherung gegen Krankheit markiert, wurde Dänemark zu einem der Vorreiter eines universalistischen Systems der sozialen Sicherheit.

\section{Alterssicherung nach dem allgemeinen System}

Mit Wirkung zum 1. Januar 1984 wurden in Dänemark die verschiedenen früheren Rentengesetze, darunter das Invaliditätsrentengesetz, in einem einzigen Gesetz zusammengefaßt, dem „Sozialrentengesetz"3. Das Rentenalter wurde für alle Bürger geschlechts- und personenstandsneutral zuerst auf das vollendete 67. Lebensjahr festgesetzt, später auf das 65 für die Personen, die nach dem 1.7.1999 das 60. Lebensjahr vollenden ${ }^{4}$. Die Voraussetzungen für das Vorliegen der Versicherteneigenschaft sind ${ }^{5}$ :

1 So Christiansen und Petersen, The Nordic Welfare States: A Historical Reappraisal, Scandinavian Journal of History, 26:3, S. 153.

2 Lov om alderdoms understøttelse til værdige trængende udenfor fattig væsenet vom 9.4.1891.

3 Lov om socialpension nr. 217 vom 16. Mai 1984. in der Fassung des Lovbekendtgørelse 2005-0802 nr. 759 om social pension; allgemein dazu s. auch Petersen, Aktuelle Reformen der Alterssicherung in Dänemark, in: $G V G$ (Hrsg.), Alterssicherung in Europa, 2007, S. 43 ff.

4 Lov nr. 287 vom 12. Mai 1999 om ændring af lov om social pension.

5 Lov om socialpension, $\S \S 2$ Abs. 1, 3 Abs. 1, 4. 
- dänische Staatsbürgerschaft,

- fester Wohnsitz in Dänemark bei Erreichen des Rentenalters,

- wenigstens drei Jahre fester Wohnsitz in Dänemark zwischen dem vollendeten 15. und 65. Lebensjahr.

Die volle Volksrente wird dann geleistet, wenn im Zeitraum zwischen dem vollendeten 15. und 65. Lebensjahr 40 Jahre mit festem Wohnsitz in Dänemark zurückgelegt worden sind; bei kürzeren Wohnsitzzeiten wird die Rentenhöhe entsprechend gekürzt.

Die meisten Leistungen der sozialen Sicherheit sind bedarfs- und/oder einkommensabhängig ausgestaltet. So wird einerseits selbst der für die Volksrenten geltende Grundbetrag um $30 \%$ des Betrags des persönlichen Einkommens gekürzt, das einen jährlich festgesetzten Freibetrag übersteigt. Andererseits haben bedürftige Volksrentner bei bestimmten Bedarfslagen Anspruch auf diverse Zulagen.

Die „Arbeitsmarkt-Zusatzrente“ (Arbejdsmarkedets Tilloegspension, ATP) ist eine auf Gesetz beruhende Altersrentenzusatzversicherung für Arbeitnehmer ${ }^{6}$. Sie wurde 1964 eingeführt, um eine zweite, beitragsbezogene Geldleistung neben der Volksrente zu schaffen. Die ATP folgt dem Kapitaldeckungsprinzip. Gesichert sind Arbeitnehmer zwischen dem 16. und 66. Lebensjahr mit wenigstens zehn Arbeitsstunden pro Woche und Wohnsitz in Dänemark. Invaliditätsleistungen sind nicht vorgesehen. Die ATP gewährt eine (Zusatz-)Altersrente für Versicherte, die das 67. Lebensjahr vollendet haben. Die Rentenhöhe beruht ausschließlich auf der Dauer der Versicherungszeit und auf den Beiträgen, die zu 2/3 von den Arbeitgebern und zu 1/3 von den Arbeitnehmern gezahlt werden.

Neben der Volksrente und der ATP hat sich auf dem dänischen Arbeitsmarkt eine Vielzahl von Modellen betrieblicher (Zusatz-)Alterssicherung auf der Grundlage von Tarifverträgen, im Einzelfall auch von Individualverträgen etabliert. Sie sind beitragsbestimmt; bei einer Beitragsbemessungsgrenze zwischen $12 \%$ und $15 \%$ der Löhne tragen die Arbeitgeber jeweils 2/3 und die Arbeitnehmer 1/3 der Beiträge.

\section{Alterssicherung der ,,Tjenestemcend“ (Beamte)}

\section{a) Das ,Beamtenstatut“"}

Mit annähernd 700.000 Beschäftigten gehören fast 1/3 aller Arbeitnehmer dem öffentlichen Dienst im staatlichen Bereich oder in den Gebietskörperschaften an. Diese Beschäftigungsverhältnisse folgen zum größten Teil (ca. 580.000) kollektivvertraglichen Regelungen; arbeits- und sozialrechtlich gelten keine Besonderheiten.

6 Lov om Arbejdsmarkedets Tillægspension (ATP) Nr. 46 vom 7. März 1964. 
Demgegenüber beruhen die ca. 55.000 Dienstverhältnisse beim Staat ${ }^{7}$ auf dem „Tjenestemanslov"8 (Beamtengesetz), die der ca. 59.000 Kommunalbeamten auf dem ,Regulativ" "9. Die Verfassung schreibt vor, die Dienstverhältnisse der Tjenestemcend durch Gesetz zu regeln. Ebenfalls verfassungsrechtlich geboten ist die Einstellungsvoraussetzung der dänischen Staatsbürgerschaft sowie die Pflicht der Beamten, einen Eid auf die Verfassung zu leisten ${ }^{10}$. Weitere wichtige Unterschiede ${ }^{11}$ zwischen Tjenestemoend und anderen Beschäftigten sind, daß

- Beamte kein Streikrecht haben,

- sie beim Wegfall der Planstelle Anspruch auf Weiterzahlung der Bezüge für drei Jahre haben,

- für sie ein besonderes, leistungsbestimmtes Alterssicherungssystem besteht.

\section{b) Die Pensionsordnungen}

Die Pensionen sind für Staats- und Kommunalbeamte gesetzlich geregelt ${ }^{12}$. Voraussetzung für Ansprüche aus beiden Pensionsordnungen - Altersrente, Dienstunfähigkeitsrente, Hinterbliebenenrenten - ist eine Wartezeit von zehn anrechnungsfähigen Dienstjahren. Die Altersrente kann mit dem vollendeten 60. Lebensjahr angetreten werden, doch erfolgen dann bestimmte Abzüge in Relation zum Diensthöchstalter von 70 Jahren ${ }^{13}$. Die Höhe der Pension wird auf der Basis des pensionsbegründenden Gehalts zum Zeitpunkt des Abschieds aus dem Dienst und dem „Pensionsalter“, das der Beamte erdient hat, errechnet. Pensionsbegründend ist das zuletzt nach der gesetzlichen Einstufung der Dienststelle erreichte Gehalt. Das „Pensionsalter“ beginnt mit dem vollendeten 25. Lebensjahr des Beamten, maximal können 37 Jahre ${ }^{14}$ erreicht werden. Die höchste Pension beträgt dann $57 \%$ des pensionsbegründenden Gehalts. Die Finanzierung erfolgt für die Staatsbeamten über den Haushalt im Umlageverfahren, für die Kommunalbeam-

7 Zahlen nach Danmarks Statistik (Hrsg.), Statistisk Årbok 2008, Tabel 257 ff.

8 Lovbekendtgørelse 2004-06-11 nr. 531 om tjenestemænd som ændret ved L 2005-06-24 nr. 527 og L 2008-06-17 nr. 484.

9 Tjenestamands regulativ af 2004 for kommuner inden for KL's forhandlingsomrade.

10 Lov 1953-06-05 nr. 169 Danmarks Riges Grundlov, § 27 Abs. 1.

11 Daneben ist noch (u.a.) die Ernennung durch den König oder den Minister, das gesetzliche Erfordernis bestimmter persönlicher Voraussetzungen (Gesundheit), Verhaltenspflichten im und außer Dienst, die Disziplinarordnung und eine bis zu zweijährige Probezeit zu nennen.

12 S. für die Staatsbeamten Lovbekendtgørelse 2004-03-19 nr. 230 om tjenestemandspension und weitgehend wortgleich für die Kommunen das Pensionsregulativ af 2008 for Tjenestamand i kommuner inden for KL's forhandlingsomrade.

13 Dies gilt nicht für Militär- oder Polizeiangehörige, deren Dienstshöchstalter erheblich niedriger festgelegt ist.

14 Nach der komplizierten Regelung des Kap. 2, § 6 tjenestemandspensionlov bzw. Kap. 2, § 6 Regulativ bewirken die verschiedenen Lebensalterstufen unterschiedliche Prozentpunkte von 1,75\% für die ersten 16 Jahre, fallend bis zu 1 \% für die letzten vier Jahre des „Pensionsalters“. 
ten im kapitalgedeckten System der „Pensionskassen“ der Kommunen. Die Pensionen unterliegen der Einkommenssteuer.

\section{Reformen}

Seit 1997 gab es ein von ATP verwaltetes obligatorisches sog. „Besonderes Rentensparprogramm" 15: Dabei wurden $1 \%$ des Lohneinkommens für eine kapitalgedeckte Zusatzrente bei Erreichen des Volksrentenalters abgeführt. 2005 wurde für die Versicherten die Möglichkeit geschaffen, selbst bei Übernahme der dafür anfallenden Kosten über das individuell angesparte Kapital zu verfügen. Das politisch kontroverse Programm wurde Ende 2007 eingestellt.

Angesichts demografischer und absehbar daraus folgender finanzieller Probleme erfolgten um die Jahrtausendwende diverse Reformen der Alterssicherung. Weil immer mehr Versicherte die von der Arbeitslosenversicherung gebotene Möglichkeit wahr nahmen, mit 60 eine vorzeitige Rente (sog. ,efterløn“16) bis zum Erreichen des Rentenalters zu beziehen, wurde, um die Laufzeit der efterløn-Leistungen zu verkürzen, das Volksrentenalter für Geburtsdaten vor dem 1. Januar 1959 auf 65 gesenkt. Für spätere Jahrgänge wird es ab 1. Januar 2009 stufenweise aber wieder auf 67 angehoben; ab 2015 soll alle fünf Jahre durch einen regelgebundenen Mechanismus überprüft werden, ob das Renteneintrittsalter der statistischen Lebenserwartung angepaßt werden muß ${ }^{17}$. Die Altersgrenzen der Kommunalbeamten entsprachen denen der Volksrente; sie wurden diesen Änderungen angepaßt ${ }^{18}$. Die Versorgung der Staatsbeamten erfuhr inhaltlich keine Reform. Die Haushaltsbelastung durch derzeit etwa 83.000 laufende Pensionen 19 wird indirekt dadurch gesenkt, daß die für Beamte vorgesehenen Planstellen reduziert ${ }^{20}$ und die Arbeitsplätze durch Angestellte auf Vertragsbasis besetzt werden. In Zukunft werden nur mehr „Männer und Frauen in Uniform“21 (Polizei, Militär, Justizvollzug und Priester der Staatskirche) den Sonderstatus eines Beamten bekleiden.

15 I. e. Særlige pensionsopsparing, SP

16 Lovbekendtgørelse 2007-08-08 nr. 994 om arbejdsløshedsforsikring m.v., Kap. 11 a. Efterløn

17 Lov om socialpension, § 1 a.

18 Regulativ, $\S \S 3$ a und 4 Abs. 1.

19 Sowie 29.000 Hinterbliebenenpension, s. Finansministeriet, Personalstyrelsen, Ansættelse i Staten, 2003, S. 30.

20 Seit 2001 wurden die Staatsbeamtenstellen auf folgende Gruppen reduziert: Cirkulære 2000-10-17 nr. $164 \mathrm{om}$ anvendelse af tjenestemandsansættelse i staten og folkekirken, § 2:1) Retsassessor.2) Politiassessor. 3) Statsadvokatassessor. 4) igsadvokatassessor. 5) Stillinger i politikorpset. 6) Vicefængselsinspektør. 7) Uniformeret personale i Kriminalforsorgen. 8) Personel af officersgruppen af linien samt auditørpersonel i forsvaret. 9) Personel af sergent- og konstabelgruppen af linien efter reglerne i kundgørelse for forsvaret B.5-28 og B.5-29. 10) Fast befalingsmand i Beredskabskorpset. 11) Fiskerikontrolpersonale.

21 Finansministeriet, Personalstyrelsen, Ansættelse i Staten (Fußn. 19), S. 31. 


\section{Norwegen}

\section{Alterssicherung nach dem allgemeinen System}

\section{a) Das bisher geltende Recht}

Die Alterssicherung sieht nach dem Folketrygdelov 22 mit der „Grundrente“ (grunnpensjon $^{23}$ ) und der „Zusatzrente“ (tilleggspensjon ${ }^{24}$ ) zwei unterschiedliche Leistungen vor. Der Kreis der Versicherten ist für beide Rentenarten gleich. Denn alle Personen, die in Norwegen leben, sind obligatorisch vom Folketrygdlov erfaßt ${ }^{25}$. Finanzierung, Leistungsvoraussetzungen und Leistungen sind jedoch unterschiedlich.

Anspruchsvoraussetzung für die Grundrente ist eine Versicherungszeit (Wohnsitz) in Norwegen von drei Jahren im Alter zwischen dem 16. und 66. Lebensjahr. Die volle Grundrente in Höhe eines „Grundbetrags“ (grunnbeløbet ${ }^{26}$ ) setzt 40 Jahre Versicherungszeit voraus, ansonsten wird die Rente prozentual entsprechend gemindert. Wer keinen oder nur einen geringfügigen Zusatzrentenanspruch hat, bekommt die „Sonderzulage“" (scertillegg), die in einem Prozentsatz zum Grundbetrag festgelegt wird ${ }^{27}$. Das gesetzliche Rentenalter ist 67, es kann bis zum 70. Lebensjahr hinausgeschoben werden. Ein Rentenbeginn ist bei entsprechender Leistungskürzung ab dem 62. Lebensjahr möglich. Hat ein Rentner weiter Erwerbseinkünfte, die den Grundbetrag übersteigen, wird die Grundrente um $40 \%$ des Betrags gekürzt, der über dem Grundbetrag liegt.

Um Ansprüche auf die Zusatzrente zu erwerben, muß zwischen dem 16. und 66. Lebensjahr über mindestens drei Jahre ein Einkommen erzielt worden sein, das den jeweils geltenden Grundbetrag übersteigt ${ }^{28}$. Dieser Betrag wird dann durch den Grundbetrag dividiert, um die jährlich erworbenen Rentenpunkte zu errechnen ${ }^{29}$. Rentenpunkte können bis zu einer Einkommensgrenze in Höhe des zwölffachen Grundbetrags erzielt werden, wobei für Einkommen zwischen dem sechs- und zwölffachen Grundbetrag Ren-

22 Lov 1997-02-28 nr. 19: Lov om folketrygd (folketrygdloven), zuletzt geändert mit Lov 2008-06-27 nr. 52.

23 Folketrygdloven Teil II, Kap. $1 \S \S 3-2$. bis 3-7.

24 Folketrygdloven Teil II, Kap. 2 § 3-8. bis 3-16.

25 Folketrygdloven Teil I, Kap. 2: Medlemskap.

26 Dieser „Grundbetrag“ wird vom Gesetzgeber (Storting) einmal oder mehrmals jährlich in Relation zum allgemeinen Einkommensniveau festgesetzt, s. Folketrygdloven Teil I, Kap. 1, § 1-4.

27 Folketrygdloven Teil I, Kap. 1, § 3-3.

28 Folketrygdlov, Teil II, Kap. 3, § 3-8.

29 Diese „pensjonspoeng“ werden auf zwei Dezimalstellen berechnet, Folketrygdlov, Teil II, Kap. 3, $\S 3-13$. 
tenpunkte nur zu einem Drittel gutgeschrieben werden ${ }^{30}$. Es können höchstens sieben Rentenpunkte jährlich erworben werden ${ }^{31}$.

Es gilt also folgende Formel (Grundbetrag $=\mathrm{G}$ ):

(Einkünfte über $\mathrm{G}$ bis Bemessungsgrenze minus ein $\mathrm{G}$ ) ./. $\mathrm{G}=$ Rentenpunkte

Die Höhe der Zusatzrente errechnet sich aus der Anzahl der Versicherungsjahre und den erworbenen Rentenpunkten. Die Vollrente setzt 40 Versicherungsjahre voraus, ansonsten wird ebenfalls proportional gekürzt. Die höchste zu erzielende Zusatzrente ist auf $42 \%$ des Betrags begrenzt, der sich ergibt, wenn der aktuelle Grundbetrag mit den während der besten 20 Jahre erzielten Rentenpunkten (,Endpunktezahl“ = sluttpoenge$t{ }^{32}$ ) multipliziert wird. Bei weniger als 20 Versicherungsjahren gilt der Durchschnitt der insgesamt erzielten Rentenpunkte als „Endpunktezahl“.

Daraus ergibt sich folgende Rentenformel:

(„Endpunktezahl“ x G x 42 x Anzahl persönlicher Versicherungsjahre) ./. (100 x 40) $=$ Rentenzahlbetrag

Das leistungsbezogene Grundrentensystem wird also mit einem beitragsbezogenen System kombiniert. Beide Renten sind einkommenssteuerpflichtig.

\section{b) Das ab dem 1. Januar 2010 geltende Recht}

Trotz einer im Vergleich zu vielen anderen europäischen Staaten hervorragenden ökonomischen Situation setzte die Regierung 2001 eine Kommission ein mit dem Auftrag, Vorschläge zur Modernisierung und Stabilisierung des gesamten Alterssicherungssystems zu erarbeiten. Im Januar 2004 lag deren Bericht vor ${ }^{33}$. Die Empfehlungen wurden im Storting diskutiert, ${ }^{34}$ schrittweise vom Gesetzgeber umgesetzt, 35 um schließlich zum 1. Januar 2010 als „Neue Alterssicherung“ (Ny alderspension) als neues Kapitel 20 in das Folketrygdelov eingefügt zu werden. ${ }^{36}$ Nach der an die Erwartungen bis zum Jahr 2050 ausgerichteten Perspektive der Reform sind die wesentlichen Punkte

- eine Flexibilisierung des Rentenalters,

- eine engere Verknüpfung zwischen Beiträgen und Leistungen,

- die mit der steigenden Lebenserwartung korrespondierende Einführung einer „Lebensalterjustierung“ durch einen demografischen Faktor in die Rentenformel

- und die Vereinfachung und Harmonisierung des bestehenden Systems.

30 Ebda.; bis 1992 lag die Bemessungsgrenze beim 8-fachen des Grundbetrags, betrifft also nicht mehr die Ehezeit.

31 Folketrygdlov Teil II, Kap. 3,§ 3-12.

32 Folketrygdlov Teil II, Kap. 3, § 3-8.

33 I. e. NOU 2004:1.

34 Stortingsmeldingen nr. 5 (2006-2007) vom 20. Oktober 2006.

35 So betreffend der betrieblichen Zusatzrenten mit Lov om obligatorisk tjenestepensjon.

36 Lov 2009-06-05-32 om endringer i folketrygdloven (ny alderspension). 
Die Alterssicherung beruht jetzt auf der „einkommensbezogenen“ (inntektspensjion) Rente und der Garantierente für Versicherte, die gar keine oder eine zu niedrige inntektspensjion beanspruchen können. ${ }^{37}$ Das neue Recht gilt voll für Personen ab dem Geburtsjahrgang 1963, für die Jahrgänge 1954-1962 ist eine schrittweise Umstellung in der Weise vorgesehen, daß zuerst 1/10 der Altersrenten nach dem neuen Recht und 9/10 nach dem alten, dann $2 / 10 \mathrm{zu} 8 / 10$, bis zum Jahrgang 1962, der 9/10 nach neuem und 1/10 nach altem Recht beziehen wird. ${ }^{38}$ Altersrente kann frühestens mit vollendetem 62. Lebensjahr bezogen werden, wobei ein Rentenbeginn vor dem 67. Lebensjahr nur möglich ist, wenn der Versicherte wenigstens das Niveau der Garantierente erreicht hat. ${ }^{39}$ Die Rentenhöhe (pensjonsbeholdning) der inntektspensjon wird vom pensionsbegründenden Einkommen 40 mit einer Begrenzung bis zum 18,1-fachen des 7,1-fachen des Grundbetrags ${ }^{41}$ bestimmt. Die Altersrente wird bei vollendetem 61. Lebensjahr ,justiert“, d.h. der individuelle Rentenanspruch wird in Relation zum durchschnittlichen Lebensalter der Bevölkerung gesetzt. ${ }^{42}$ Dies erfolgt durch die „Teilungszahl“ (delingstall), eine mathematische Formel, in der diese Relation der individuellen und allgemeinen Lebenserwartung enthalten ist. ${ }^{43}$ Die Höhe der Renten wird jährlich zum 1. Mai der Lohnentwicklung angepaßt. ${ }^{4}$

\section{c) Finanzierung}

Im Folketrygdlov ist die gesamte soziale Sicherheit kodifiziert. Neben der Alterssicherung werden Geld- und Sachleistungen bei Invalidität, Krankheit, Unfall, Arbeitslosigkeit geregelt, sowie Familien- und Pflegeleistungen, Elterngeld, Sozialhilfe bis hin zur Finanzierung all dieser Leistungen durch Beiträge der Arbeitnehmer, Arbeitgeber und Selbständigen und durch Zuschüsse des Staates. Zusammen sollen die Beiträge und der Staatszuschuß die gesamten Jahresausgaben aufgrund des Folketrygdlov ausgleichen 45 . Der Staatszuschuß allein soll mindestens die Ausgaben für die Grundrente (und bestimmter anderer Sozialleistungen) sowie der neuen Garantierente decken ${ }^{46}$. Die Finanzierung dieses Teils der Alterssicherung erfolgt weitgehend durch den „Staatlichen

37 Ebda., § 20-3.

38 Ebda., § 20-1 i.V.m. § 20-19.

39 Ebda., § 20-2 i.V.m. § 20-15.

40 Wozu auch Wehrdienst- und Elternurlaubszeiten und Zeiten der Pflege zählen sowie Zeiten mit Bezug von Krankengeld, Lov 2009-06-05-32, § 20-4 i.V.m. § 20-6 bis § 20-8.

41 Lov 2009-06-05-32, § 20-4.

42 Ebda., § 20-12.

43 Ebda., § 20-13.

44 Unter Abzug von 0,75 \%, Lov 2009-06-05-32, § 20-18.

45 Folketrygdelov, Teil VIII, Kap. 23, § 23-1.

46 Ebda., § 23-10. 
Pensionsfonds“47, dem früheren staatlichen Erdölfonds, der die Rentenausgaben ,auf lange Sicht durch Einsatz der staatlichen Petroleumeinkünfte“ tragen soll ${ }^{48}$. Die Zusatzrente wird allein aus den Versichertenbeiträgen zum Folketrygdlov finanziert. Der Arbeitgeberbeitrag beträgt nach geographischen Regionen abgestuft von $0 \%$ bis höchstens $14,1 \%$ der Lohnsummen ${ }^{49}$. Arbeitnehmer zahlen ab einem bestimmten Freibetrag 7,8 \% des rentenbegründenden Einkommens, Selbständige 10,7 \% bis zu Einkünften in Höhe des zwölffachen Grundbetrags. In keinem Fall sollen die Beiträge $25 \%$ der über dem Freibetrag liegenden Einkünfte übersteigen.

Daneben entwickelten sich für eine wachsende Zahl von Arbeitnehmern steuerlich begünstigte Betriebsrenten, die meist von Arbeitgebern und Arbeitnehmern gemeinsam durch Beiträge finanziert werden. Zum 1. Januar 2006 wurde die Einrichtung betrieblicher Alterssicherung für die meisten Arbeitgeber obligatorisch 50 .

\section{Alterssicherung der ,,Tjenestemenn“(Beamte)}

\section{a) Das ,Beamtenstatut“}

Für die Dienstverhältnisse von Arbeitnehmern „,im Dienste des Staates“ gilt das „Gesetz betr. die Staatsbediensteten“ (,tjenestemennlov“51, Beamtengesetz). Als „,jjenestemann“ gilt jeder Arbeitnehmer im Staatsdienst, der nicht „embetsmann“ ist.

Der Rechtsstellung der embetsmenn (deutsch wörtlich „Amtmann“) gelten mit Blick auf das Staatsganze zunächst mehrere Artikel der Verfassung (grunnlov ${ }^{52}$ ): So wird über die norwegische Staatsbürgerschaft hinaus auch verlangt, daß die norwegische Sprache beherrscht wird ${ }^{53}$ und weitere „die Verbindung zum Land sichernde Bedin-

47 Lov-2005-12-21 nr. 123: Lov om Statens pensjonsfond. Er besteht aus dem „Statens pensjonsfond Utland“ und dem „Statens pensjonsfond - Norge“, der als „Folketrygdfond” mit eigener Rechtspersönlichkeit separat verwaltet wird, Lov 2007-06-29 nr. 44: Lov om Folketrygdfondet.

48 Lov 2007-06-29 nr. 44, § 1: Statens pensjonsfond skal understøtte statlig sparing for finansiering av folketrygdens pensjonsutgifter og underbygge langsiktige hensyn ved anvendelse av statens petroleumsinntekter.

49 Folketrygdlov, Teil VIII. Kap. 23, § 23-2. Der Höchstbeitrag ist in Oslo zu zahlen, bei Beschäftigung im Norden des Landes wird kein Beitrag erhoben.

50 Lov 2005-12-21 nr. 124 om obligatorisk tjenestepensjon. Danach ist schon ab zwei Angestellten mit $75 \%$ der vollen Arbeitszeit die Einrichtung einer beitragsbezogenen ,tjenestepensjon“ obligatorisch.

51 Lov 1983-03-04 nr. 03: Lov om statens tjenestemenn m.m.

Zum Verwaltungsrecht in Norwegen Sperr, Verwaltungsrechtsschutz in Deutschland und Norwegen, 2009. In dieser Arbeit wird ,innerhalb der vergleichend-analysierenden Auswertung der funktionale Kontext nationaler Eigenheiten beachtet, funktionale Äquivalente werden einbezogen." (S. 31).

52 Lov 1814-05-17 nr. 00: Kongeriget Norges Grundlov, given i Rigsforsamlingen paa Eidsvold den 17de Mai 1814.

53 Grundlov $\S 92$. 
gungen“54 erfüllt sind. Diesem Ziel gelten auch $\S 21$, wonach der König die embetsmenn beruft und diese auf Verfassung und Könige vereidigt werden, ${ }^{55}$ und $\S 28$, wonach die Besetzung hoher Ämter wie ,andere wichtige Angelegenheiten dem Staatsrat vorzutragen sind“"56. Der Gesetzgeber hat aber weitgehend freie Hand, welche Stellen er mit embetsmenn besetzen will ${ }^{57}$. Eine Entfernung aus dem Amt kommt nach $\S 22$ grundsätzlich nur durch Gerichtsurteil in Betracht ${ }^{58}$. Daneben fallen die embetsmenn dann unter das Beamtengesetz, wenn dies darin ausdrücklich bestimmt wird ${ }^{59}$.

Nach diesem Gesetz werden alle Staatsbedienstete grundsätzlich unbefristet eingestellt, die Ausnahmen sind ebenso im Gesetz geregelt ${ }^{60}$ wie die Einstellungsmodalitäten der Bediensteten61. Für tjenestemenn ist eine Probezeit von sechs Monaten vorgeschrieben 62 . Dienstverhältnisse von tjenestemenn und embetsmenn können nur nach den engen Vorgaben des Gesetzes beendet werden 63 . Vor einer Kündigung soll bei allen Beamten geprüft werden, ob nicht eine andere dienstliche Verwendung in Betracht kommt; ist dies nicht sofort möglich, kann für bis zu drei Jahren ein „Wartegeld“ gezahlt werden ${ }^{64}$. Das Gesetz enthält auch Regeln für eine Disziplinarordnung, zur Suspendierung vom Dienst und für den Rechtsweg bei Beschwerden ${ }^{65}$. Allen Beamten ist die Annahme von Geschenken, Provisionen, etc. verboten ${ }^{66}$.

54 So bezüglich der weiteren Bedingungen wie etwa Geburtsort in Norwegen Eckhoff/Smith, Forvaltningsrett, 2006, S. 359.

55 Grundlov $\S 21$ : Kongen vælger og beskikker, efter at have hørt sit Statsraad, alle civile, geistlige og militære Embedsmænd.

56 Grundlov §. 28: Forestillinger om Embeders Besættelse og andre Sager af Vigtighed skulle foredrages i Statsraadet.

57 Grundlov $\S 22$ nennt nur das Amt der Staatsräte, Bischöfe, höherer Offiziere und einige andere, die mit embetsmenn besetzt werden müssen.

58 Grundlov §. 22. Es gibt bislang nur ein Fallbeispiel aus dem Jahr 1984 (Sache Treholt), daß ein Beamter aus dem Außenministerium wegen Spionage nach $\S 22$ des Amtes enthoben wurde, s. Andences und Fliflet, Statsforfatningen i Norge, 10. Aufl. 2006, S. 338.

59 Tjenestemennlov Kap. $1, \S 1$ S. 2.

60 Ebda., $\$ 3$.

61 Ebda., § 4-6.

62 Tjenestemennlov Kap. $2, \S 8$.

63 Ebda., §§ 9-13.

64 Ebda., $§ 13$.

65 Ebda., $\S \S 14-19$.

66 Tjenestemennlov Kap. 3, § 20. 


\section{b) Die Pensionsordnungen}

Die Alterssicherung der staatlichen und der meisten kommunalen ${ }^{67}$ Beamten ist im „Gesetz betr. die Staatliche Pensionskasse“ (Statens Pensjonskasse, SPK) ${ }^{68}$ geregelt. Die kommunalen Pensionsordnungen folgen inhaltlich weitgehend diesem Gesetz ${ }^{69}$.

Bei der SPK handelt es sich um eine ,staatsgarantierte Kasse zur Deckung der Pensionen"70, die Mittel verwaltet das Finanzministerium. Mitglieder der Kasse sind Staatsbedienstete mit wenigstens 14 Arbeitsstunden/Woche ${ }^{71}$. Um Mitglied werden zu können, müssen auch die Voraussetzungen des trygdelov erfüllt sein, primär also Wohnsitz in Norwegen und wenigstens drei Dienstjahre. Für die Zusatzrente, die auch für die Beamten obligatorisch ist, sind Beiträge aus dem Teil der Bezüge zu leisten, die für die Pensionskasse als pensionsbegründendes Einkommen gelten ${ }^{72}$. Das ist das Einkommen aus dem Dienstverhältnis, das zur Mitgliedschaft bei der SPK berechtigt ${ }^{73}$, begrenzt auf das zwölffache des Grundbetrags nach dem trygdelov ${ }^{74}$. Aus diesem Einkommen (,Pensjonsgrunnlaget“, Pensionsgrundlage) werden vom Dienstherrn $2 \%$ als Beitrag des Versicherten an die SPK einbehalten und abgeführt ${ }^{75}$. Die Altersgrenzen für die Pension differieren ja nach Dienststelle; obwohl sie generell bei 70 Jahren liegt $^{76}$, besteht doch Anspruch auf Pension ab dem 67. Lebensjahr ${ }^{77}$. Nach der sog. „85-JahreRegel“78 kann ein Versicherter drei Jahre vor der für ihn geltenden Altersgrenze dann in Pension gehen, wenn seine Dienstzeit addiert mit dem Lebensalter 85 ergibt $^{79}$. Ein

67 Daneben finden sich noch bei einigen Kommunen eigene Versorgungskassen, andere Gemeinden haben sich zu einer Versicherung zusammengeschlossen, etwa der Kommunal Landspensjonskasse (KPL). Auch für Seeleute und Fischereiaufsicht gibt es eigene öffentliche Pensionskassen, i. e. Pensjonstrygden for sjømenn und Fiskerpensjonsordningen, s. Arbeids- og velferdsforvaltningen (NAV), Tjenestepensjoner, www.nav.no.

68 LOV 1949-07-28 nr. 26: Lov om Statens Pensjonskasse.

69 S. Kjønstad / Syse, Velderdsrett I., 3. Aufl. 2006, S. 35.

70 Lov om Statens Pensjonskasse Kap. 1, $\S 1$ : „Statens Pensjonskasse er en tatsgaranterert kasse til dekning av de pensjoner"

71 Lov om Statens Pensjonskasse Kap. 2, § 5; hinsichtlich der Arbeitszeit gibt es Ausnahmen, etwa für Lehrer.

72 Lov om Statens Pensjonskasse Kap. 2, § 6.

73 Ebda.,, Kap. $3 \S 11$.

74 Ebda., $§ 14$.

75 Ebda., $\S \S 16$ und 17.

76 Lov 1956-12-21 nr. 01: Lov om aldersgrenser for offentlige tjenestemenn m. fl., § 2; wenn der Dienst besondere Anforderungen stellt, kommt eine Reduzierung auf 68, 65, 63 und 60 Jahre in Betracht. Eine Verlängerung der Dienstzeit um zwei Jahre ist einmal möglich, dann sind nur mehr einjährige Verlängerungen um zusammen höchstens fünf Jahre vorgesehen, § 3 .

77 Lov om Statens Pensjonskasse Kap. 5, § 21.

78 Ebda., § 21 Abs. 2.

79 Danach könnte ein Polizeibeamter, dessen Altersgrenze bei 60 Jahren liegt, mit 57 abschlagsfrei in Pension gehen, wenn er 28 Dienstjahre zurückgelegt hat, Beispiel nach Norges offentlig utredninger (NOU) 2004:1: Moderniserert folketrygd, Bærekraftige pensjon for framtida, S. 310. 
Anspruch auf die volle Alterspension in Höhe von $66 \%$ des zuletzt bezogenen pensionsbegründenden Gehalts ${ }^{80}$ setzt 30 Dienstjahre voraus, bei weniger Dienstjahren wird um jeweils 1/30 gekürzt. ${ }^{81}$ Für die Pensionen besteht eine sog. „Bruttogarantie“ ${ }^{42}$, d.h., daß alle individuell erworbenen Alterssicherungsleistungen auf den Zahlbetrag der höchst möglich zu erreichende Pension begrenzt werden. Pensionen aus der SPK werden gegebenenfalls um 3/4 des Grundbetrags aus dem folketrygdlov gekürzt ${ }^{83}$. Da die auf das pensionsbegründende Einkommen bezogenen Beiträge zur Zusatzrente höhere Ansprüche ergeben und diese bei der gesetzlichen ,Zusammenrechnung“ (samordning) nicht berücksichtigt werden, erreichen viele Beamte aufgrund dieses „Fiktivvorteils“ 84 höhere Altersbezüge als die vom Gesetz vorgegebenen $66 \%$ des Letztgehalts plus $1 / 4$ Grundbetrag. Die laufenden Pensionen sind über den Bezug auf den Grundbetrag dynamisiert. Sie zählen zum steuerpflichtigen Einkommen.

\section{Reformen}

Bezüglich der tjenestepensjon hat die Regierung im Mai 2005 bekräftigt, daß es bei einer an 2/3 des Letztgehaltes ausgerichteten Pension bleiben werde ${ }^{85}$. Durch die Reform der allgemeinen gesetzlichen Alterssicherung sollen die Pensionen des öffentlichen Dienstes nicht gekürzt werden. Eine Anpassung an das allgemeine System sei nur hinsichtlich der „,delningstall“ vorgesehen. Wann und wie dies durchgeführt werden soll, wird in enger Zusammenarbeit mit den Tarifpartnern des öffentlichen Dienstes erarbeitet werden.

80 Lov om Statens Pensjonskasse Kap. 5, § 22. Hatte ein Beamter in früheren Jahren ein höhere Einstufung als bei Erreichen des Pensionsalters, so soll das höhere Gehalt der Pensionsberechnung zugrunde gelegt werden, s. ebda., Kap. 3, § 15 Abs. 2.

81 Lov om Statens Pensjonskasse Kap. 5 § 23. Als „Dienstjahre” gelten die Jahre der Mitgliedschaft in der Pensionskasse, s. ebda., Kap. 4, § 19.

82 LOV 1957-07-06 nr. 26: Lov om samordning av pensjons- og trygdeytelser; zu dessen Hintergründen s. NOU 1995:29: Samordning av pensjons- og trygdeydelser.

83 Ebda., § 19.

$84 \mathrm{Zu}$ diesem „fiktivfordel“ und zur progressiven Besteuerung der Pensionen NOU 2004:1, S. 310-311.

85 Information des Arbeids- og Inkluderingsdepartement, Pensjonsreformen (14.7.2008) www.regjeringen.no, S. 9-11. 


\section{Schweden}

\section{Alterssicherung nach dem allgemeinen System}

Die Alterssicherung war zum Jahr 2000 in Schweden Gegenstand einer tiefgreifenden Strukturreform: Die traditionsreiche steuerfinanzierte Volksrente wurde durch eine beitragsfinanzierte „einkommensbezogene Rente“ (inkomstgrundade ålderspension) ${ }^{86}$ ersetzt, ergänzt durch eine kapitalgedeckte „Prämienrente“ (premiepension) ${ }^{87}$ und die „Garantierente“ (garantipension) ${ }^{88}$ als steuerfinanzierte Absicherung für alte Menschen, die keine hinreichenden Rentenanwartschaften verdient haben. Es handelt sich um ein geschlossenes System: Die eingezahlten Beiträge müssen vollständig vom Staatshaushalt getrennt verwaltet werden. Mit den Beiträgen eines Jahres - das sind $18,5 \%$ der Erwerbseinkünfte bis zu einer Beitragsbemessungsgrenze von 7,5 des „Einkommensgrundbetrags“" 89 abzüglich der 2,5\%, die in die Prämienversicherung gehen müssen die im selben Jahr auszuzahlenden Renten finanziert werden. Die individuellen Ansprüche werden zum 65. Lebensjahr mittels einer „Teilungszahl“ (delningstal) errechnet, die auch einen demografischen Faktor enthält. Damit wird erst die Zukunft zeigen, wie hoch die individuelle Rente jeweils sein wird. Das gesetzlich geregelte $\mathrm{Zu}-$ sammenspiel von Durchschnittseinkommensindexierung des individuellen Rentenertrags mit dem „Folgeindex“ der Rentenberechnung bei Rentenantritt gibt dem System ein hohes Selbstregulierungsvermögen gegenüber demografischen und wirtschaftlichen Veränderungen. Gerät die Balance zwischen Einkünften und Schulden des Systems dennoch ins Ungleichgewicht, so wird die in der Rentenformel enthaltene „Balancezahl“ (balanstal), also der Quotient aus Einnahmen und erworbenen Rentenansprüchen, kleiner als eins. Sobald dies eintritt, wird bei der Rentenformel automatisch vom „Durchschnittseinkommensindex“ (snittindex) zum „Summenindex“ (summaindex) gewechselt. Das bedeutet, daß die während dieser Phase erworbenen Rentenerträge in Relation zu der sehr viel langsamer (oder gar auch nicht) wachsenden Gesamtsumme aller Erwerbseinkünfte gesetzt und dadurch ,gebremst“" werden. Renten können frühestens ab dem 61. Lebensjahr bezogen werden, ein Renteneintrittsalter gibt es nicht mehr.

86 Lag (1998:674) om inkomstgrundade ålderspension.

87 Ebda., III. Abteilung, Premiepensionssystemet.

88 Lag (1998:702) om garantipension.

89 Lag (1998:674) om inkomstgrundade ålderspension Kap. 1, § 6. Dabei handelt es sich um einen Faktor der Rentenformel, der, von der Regierung jährlich festgesetzt, die allgemeine Einkommensentwicklung (mit einjähriger) Verzögerung spiegelt. 
In Kap. 11 der Verfassung (Regeringsformen ${ }^{90}$, RF) finden sich eine Reihe von Vorgaben zur Regelung des Dienstrechts der Richter und Beamten.

So schreibt RF 11: 1 vor, daß in die höchsten Gerichte nur Richter berufen werden dürfen, die Richter der ordentlichen Gerichtsbarkeit sind oder waren ${ }^{91}$. RF 11: 9 betrifft die allgemeine Anstellungskompetenz und die Anstellungsvoraussetzungen. Im ersten Satz wird bekräftigt ${ }^{92}$, daß allein der Regierung oder von ihr dazu bestimmten Behörden die Kompetenz zukommt, Beamte einzustellen. Dabei sollen nur sachliche Gründe wie „Verdienst und Eignung"93 entscheiden. In der Praxis wird insbesondere hinsichtlich Beförderungen „Verdienst“ meist mit Dienstalter gleichgesetzt ${ }^{94}$. Sodann ${ }^{95}$ werden die Dienststellen aufgeführt - darunter die der Richter und der Chefs bestimmter Behörden -, die nur mit schwedischen Staatsbürgern besetzt werden dürfen. Schließlich heißt es in RF 11:10, daß die für die Rechtstellung der Staatsbediensteten grundlegenden Bestimmungen durch Gesetz getroffen werden müssen. Dem entspricht das „Gesetz betr. die öffentliche Anstellung“96. Es unterscheidet nicht mehr nach Staats- und Kommunalbeamten oder nach Angestellten und Arbeitern. Einstellungsvoraussetzung sind in wörtlicher Übernahme des Verfassungstextes nur Eignung und Verdienst ${ }^{97}$. Über die Vorgaben der Verfassung hinaus wird für Polizei und Militär die schwedische Staatsbürgerschaft vorgeschrieben, für weitere im Gesetz angeführte Dienststellen kann die Regierung dies bestimmen ${ }^{98}$. Die Bedingungen zur Aufnahme von Nebentätigkeiten sind genau geregelt ${ }^{99}$, zur Beendigung von Dienstverhältnissen gibt es einige das Kündigungsschutzgesetz ${ }^{100}$ ergänzende Regeln ${ }^{101}$. Das Gesetz enthält auch eine „Diszipli-

90 Regeringsformen (1974:152), RF.

91 RF 11:1Abs. 1 S. 2: "...endast den tjänstgöra såsom ledamot som är eller har varit ordinarie domare i domstolen." Deren Unabsetzbarkeit ist in RF 11:5 geregelt.

92 RF 11:9 bestätigt insoweit RF 1:6: Regeringen styr riket. (=Die Regierung steuert das Reich); s. dazu und zum folgenden ausführlich Holmberg u.a., Grundlagarna. 2. Aufl. 2006, RF 11:9, S. 502 ff.

93 RF 11:9, S. 2: „endast vid sakliga grunder, såsom förtjänst och skicklighet.”

94 Holmberg u.a., Grundlagarna (Fußn. 92), S. 503; Strömberg/Lundel, Allmän förvaltningsrätt, 24. Aufl. 2008, S. $42 \mathrm{ff}$.

95 RF 11:9 S. 3.

96 Lag (1976:600) om offentlig anställning, LOA. Daneben regelt Lag (1994:261) om fullmaktsanställning primär das Dienstrecht der Richterschaft.

97 LOA $\S 4$.

98 LOA $\S 4.5,6$.

99 LOA $\S 7-7 \mathrm{~d}$.

100 Lag (1982:80) om anställningsskydd.

101 LOA $\S$ 8-13. Zum öffentlichen Dienst in Schweden s. Jägerskiöld, Schweden, in: Kaiser/Mayer/Ule (Hrsg.), Recht und System des öffentlichen Dienstes. Studienkommission für die Reform des öffentlichen Dienstes Bd. 2, 1973, S. 19 ff., und speziell zur Alterssicherung der Beamten 
narordnung"102, Vorschriften für regelmäßige Gesundheitsuntersuchungen 103 sowie Regeln für das Verhalten bei Arbeitskonflikten ${ }^{104}$. Grundsätzlich sind den Beamten Arbeitskampfmaßnahmen erlaubt, die Vorschriften haben aber kaum praktische Bedeutung, da die Tarifpartner sich auf ein schiedsgerichtliches Verfahren geeinigt haben, um Arbeitskämpfe möglichst zu vermeiden 105 .

\section{b) Die Pensionsordnung}

Das LOA überläßt die meisten Details des Dienstrechts tarifvertraglichen Regelungen, so auch die Beamtenversorgung. Dies gilt auch für die Alterssicherung, für die seit Januar 2003 der „Pensionsvertrag für Arbeitnehmer beim Staat (PA 03)“106 gilt. Tarifpartner sind für den Staat das „Arbetgivarverket“ ${ }^{\prime 107}$, für die Bediensteten der $O F R^{108}$, das gemeinsame Organ der Gewerkschaften zur Zusammenarbeit im öffentlichen Dienst, $S A C O^{109}$, ein Verbund aus 24 selbständigen Gewerkschaften sowie $S E K O$, der Gewerkschaft für Service und Kommunikation ${ }^{110}$. PA 03 gilt für Staatsangestellte ab dem 18. Lebensjahr mit mindestens $20 \%$ Beschäftigungszeit, die das Pensionsalter bei Einstellung noch nicht erreicht haben, noch keine Dienstrente beziehen und $1943^{111}$ oder später geboren sind ${ }^{112}$. Es sind zwei Pensionsleistungen vorgesehen, die „beitragsbestimmte“ (avgiftsbestämda ålderspension) ${ }^{113}$ und die „leistungsbestimmte“ (förmånsbetämda ålderspension) ${ }^{114}$ Alterspension. Das Pensionsalter liegt für beide gleich bei Erreichen des 65 . Lebensjahrs ${ }^{115}$, eine vorzeitige Pension ist ab 61 bei entsprechender Kürzung möglich, dabei kann auch eine „temporäre Pension“ bis zum Erreichen des Pensionsalters gewählt werden ${ }^{116}$.

Bramstång/Joheman, Landesbericht Schweden, in: Zacher/Bullinger/Igl (Hrsg.), Soziale Sicherheit im öffentlichen Dienst in der Bundesrepublik Deutschland, den Niederlanden, Schweden, Belgien und den Europäischen Gemeinschaften, 1982, S. 177 ff.

102 LOA $\S \S 14-22$. $\S 34$ verweist bei Disziplinstreitigkeiten auf den Beschwerdeweg zum „statlig ansvarsnämnd“, einem besonderen Schiedsgericht.

103 LOA $\S 30$.

104 LOA § 23-29.

$105 \mathrm{Zu}$ diesem „särskild statstjänstenämnden“ s. Strömberg/Lundell, Allmän förvaltningsrätt (Fußn. 94), S. 45.

106 Pensionsavtal för arbetstagere hos staten m. fl. PA 03.

107 Förordning (1976:1021) om statliga kollektivavtal, m. m.

108 Offentliganställdas Förhandlingsråd.

109 Sveriges Akademikers Centralorganisation.

110 Facket för service och kommunikation.

111 Geburtsjahrgänge vor 1948 erhalten Altersrente nach dem Pensionsvertrag von 1991, PA 91.

112 PA $03 \S 2$.

113 Ebda., $§ 5$ und Kap. 3.

114 Ebda., § 5 und Kap. 4-7.

115 Ebda., $\S 5$ für Piloten und Berufsoffiziere beträgt das Pensionsalter 60 .

116 Ebda., §§ 12, 21. 
Für die beitragsbestimmte Alterspension wird ab dem 23. Lebensjahr des Versicherten monatlich ein Beitrag von 2,5\% des Gehalts bis zu einer Bemessungsgrenze eines Jahresgehalts in Höhe des 30-fachen des Einkommensgrundbetrags des allgemeinen Systems eingezahlt ${ }^{117}$. Der Versicherte kann selbst wählen, wie diese Beiträge angelegt werden sollen ${ }^{118}$. Mit der Organisation der Wahl wurde das "Staatliche Pensionsamt“ (Statens Pensionsverket, SPV) beauftragt ${ }^{119}$. Zur Wahl stehen die Versicherungsgesellschaften, die mit dem Arbetgivarverket einen entsprechenden Vertrag abgeschlossen haben. Dem Versicherten wird ein „Wahlpaket“ mit Informationen über die Anlagemöglichkeiten, einem Wahlblankett nebst Antwortkuvert nach Hause geschickt; die Wahl, für die man zwei Monate Zeit hat, ist nur mit diesem Formular gültig. Zur Wahl stehen die Sparform, traditionelle Versicherung oder Anlagefonds, letztere mit und ohne Rückzahlungsschutz, und die jeweilige Versicherungsgesellschaft. Die Entscheidung kann jedes Jahr geändert und neu getroffen werden. Über die unterschiedlich hohen Verwaltungsabgaben, die Art der angebotenen Fonds und deren frühere Erträge etc. gibt auf Nachfrage SPV Auskunft. Die gewählte Anlage erfolgt dann von SPV für den Versicherten. Wird keine Wahl getroffen, werden die Beiträge von SPV in einer traditionellen Versicherung mit Rückzahlungsschutz angelegt.

Zusätzlich zur beitragsbestimmten Altersrente besteht nach der für diese geltenden Voraussetzungen eine „komplettierende Alterspension“120. Der Arbeitgeber führt für diese sog. „Kåpan Tjänste“ 2 \% des Gehalts an die „Kåpan Pensioner försäkringsförening“ ab, die Verwaltung erfolgt durch SPV. Ein Anlagewahlrecht des Versicherten besteht nicht. Diese kapitalgedeckte Versicherung sieht Alterspension und Hinterbliebenenleistungen vor.

Die leistungsbestimmte Alterspension ${ }^{121}$ gilt für Bedienstete mit Gehältern über dem 7,5-fachen Einkommensgrundbetrag. Die Höhe der Pension beträgt für ein Gehalt bis zur Grenze des 20-fachen Grundbetrags $60 \%$, für Gehaltsanteile vom 20- bis zum 30fachen des Grundbetrags $30 \%$. Die volle Leistung setzt 30 Dienstjahre zwischen dem 28. Lebensjahr und dem Pensionsantritt voraus. Das pensionsbegründende Gehalt umfaßt das Grundgehalt nebst diversen ständig gewährten Zulagen, die Pensionshöhe beruht auf dem Durchschnittsgehalt der letzten fünf Jahre vor Pensionsantritt.

Die Finanzierung aller drei PA 03-Pensionen erfolgt allein durch den Arbeitgeber, der für die Versicherten die Beiträge bezahlt ${ }^{122}$. Die Auszahlung der Leistungen obliegt dem SPV ${ }^{123}$, sie sind einkommenssteuerpflichtig.

117 Ebda., §9.

118 Wahlmöglichkeit und das Procedere wurde im Verhandlungsprotokoll zum PA 03 festgelegt, s. Förhandlingsprotokoll 2202-06-28, Dnr 0206-0324-Fe-41, Valet av individuell ålderspension enligt PA 03.

119 Förhandlingsprotokoll (Fußn. 118), PA $03 \S 1$. Die folgende Darstellung beruht auf der Broschüre SVP (Hrsg.), Pensionsfakta, PA 03 Tjänstepension, 2006.

120 PA $03 \S 10$ i.V.m. $\S 6$ punkt 2 und Pensionsfakta. PA 03, S. 4.

121 PA 03 Kap. 4.

122 PA 03 § 6 i.V.m. Förordning (1997:9089) om premier för statens avtalförsäkringar. 
Für Bedienstete, die aus dem Staatsdienst ohne eigenes Verschulden vier Jahre vor Erreichen des Pensionsalters ausscheiden, sieht die kollektive „Sicherheitsvereinbarung“ (Trygghetsavtalet, TA ${ }^{124}$ ) zwischen Staat und Gewerkschaften eine „Pensionsersatzleistung“ (pensionsersättning ${ }^{125}$ ) bis zum Erreichen des Pensionsalters vor. Bei Vorliegen besonderer Umstände kommt eine „besondere Pensionsersatzleistung“ (särskild pensionsersättning ${ }^{126}$ ) in Betracht. Wenn sich für einen Bediensteten die ATP-Rente nach dem (alten ${ }^{127}$ ) allgemeinen System deshalb verringert, weil für die Zeiten dieser Ersatzpensionen keine ATP-Punkte erzielt werden, so wird eine „Pensionszulage“ (pensionstillägg ${ }^{128}$ ) in Höhe der Differenz zu dem ATP-Betrag gezahlt, der bei Punkteanrechnung hätte erreicht werden können.

\section{Reformen}

Auch die neue Prämienrente ist für alle Arbeitnehmer und Selbständigen obligatorisch; hinsichtlich der Berechnungsgrundlagen der Beitragsseite (2,5\% des Gesamtbeitrags von $18,5 \%$ ) beruht sie auf den auch für die Einkommensrente geltenden sozialrechtlichen Regeln. Der Versicherte bestimmt über die Anlage seiner Beiträge und kann in gewissen Grenzen auch über die damit verdienten Ansprüche entscheiden, etwa um seinen Lebenspartner eine anteilige Rente zukommen zu lassen. Das Gesetz legt für das neue Prämienrentensystem fest,

„daß die Mittel, die für das Prämienrentenrecht festgestellt werden, in einem Fond eingebracht werden,

daß der Einzelne die Möglichkeit hat, selbst über die Verwaltung dieser Mittel auf seine Rechnung zu bestimmen, und

daß die Höhe der Rente auf der Wertentwicklung der fondierten Mittel beruht““. 129

Für die Prämienrenten wurde mit der „Prämienrentenbehörde“ (Premiepensionsmyndigheten, PPM), eine eigene Verwaltungseinheit unter der Aufsicht des Finanzministeriums gegründet, die ausdrücklich nach versicherungsmäßigen Prinzipien arbeiten soll ${ }^{130}$ und für jeden Versicherten (das Gesetz spricht in diesem Teil stets vom ,pre-

123 Förordning (2002:869) om utbetalning av statliga tjänstepensions- och grupplivförmåner.

124 Trygghetsavtalet TA i lydelse fr. o. m. den 1 januari 2008.

125 Ebda., $§ 14$.

126 Ebda., § 15.

$127 \mathrm{Zu}$ diesem s. zuletzt Köhler, Die Reform der Alterssicherung in Schweden, in: Becker/Kaufmann/von Maydell/Schmähl/Zacher (Hrsg.), Alterssicherung in Deutschland, 2007, S. 691-712; Köhler, Die Rentenreform 1957 und die Allgemeine Zusatzrente in Schweden, Deutsche Rentenversicherung 62 (2007) 2/3, S. 82-98.

128 TA $\S 18$.

129 LIP Dritter Abschnitt, Kap. 7, § 1, Übersetzung vom Verf.

130 Ebda., $\S 2$. 
miesparare“, also vom „Sparer“) das „premiepensionskonto“ führt. Während des Jahres, da die Prämienbeiträge zunächst abgeführt werden, werden diese auf einem Konto der Reichsschuldenverwaltung (Riksgäldkontoret) angesammelt und von der neuen Behörde „mit niedrigem Risiko und unter Berücksichtigung der Liquidität“131 auf dem Kapitalmarkt angelegt und zusammen mit den Zinsen am Jahresende dem nun individuell errechneten Konto des Versicherten gutgeschrieben. Dabei ist der Versicherte auf seine Wahlfreiheit hinsichtlich der Anlage der Gelder hinzuweisen. Seither haben die Versicherten jedes Jahr zu entscheiden, wo die Gelder langfristig angelegt werden sollen. Dies ist der PPM mitzuteilen, die dann die entsprechenden Transferierungen vornimmt. Dafür kommen alle (auch ausländische) bei der neuen Behörde nach den speziellen Vorgaben des Gesetzes angemeldeten 132 Wertpapierfonds in Betracht sowie der öffentlich-rechtliche „Allgemeine Prämiensparfonds“, in den immer dann von Amts wegen investiert wird, wenn der Versicherte eine eigene Wahl nicht trifft. Unabhängig davon, ob eine individuelle Wahl stattfindet oder die Behörde für den Versicherten tätig wird, trägt doch dieser allein das Risiko dafür, ob seine Prämienrentenbeiträge langfristig Gewinn bringen oder nicht. Für die Mittelverwaltung sind zahlreiche ins Detail gehende Regelungen (im Gesetz und durch Verwaltungsrichtlinien) getroffen worden, z.B. hinsichtlich der Gebühren, wenn von einem Fonds in einen anderen gewechselt werden soll u. ä.; zu Schadensersatzprozessen aufgrund schlechter Fondsverwaltung ist primär die neue Behörde befugt, der Versicherte erst, wenn diese eine Klage nicht erhebt ${ }^{133}$.

Die PPM hatte erhebliche Anlaufschwierigkeiten. Ab dem Jahr 2000 legten dann aber die ersten 4,4 Mio. Versicherten 2,5 \% ihrer Beiträge auf dem Kapitalmarkt an eine Zahl, die jährlich um etwas über 100.000 wächst. Bei voller Funktion des neuen Systems werden die Anlagekonten von etwa 7 Mio. Versicherten zu verwalten sein.

Die Prämienrente kann frühestens mit dem Monat angetreten werden, in dem der Versicherte das 61. Lebensjahr vollendet hat. Das Kapital wird nicht als „lump sum“ ausgezahlt. Es wird eine lebenslange Rente geleistet, die aufgrund des Guthabens zum Rentenantritt von der PPM errechnet wird. Der Versicherte kann eine Leibrente mit garantierter Höhe beanspruchen - dann trägt die PPM die Verantwortung (und das Risiko) dafür, daß das angesparte Guthaben des Versicherten für die einmal bestimmte Leistungshöhe ausreicht ${ }^{134}$. Es ist auch möglich, die Rente zu 1/4, $1 / 2$ oder 3/4 abzurufen und das verbleibende Kapital weiterhin anzulegen. Ehegatten können gemeinsam ihre Guthaben unter bestimmten Voraussetzungen einander gutschreiben lassen 135 .

Mit der Prämienrente nehmen alle Versicherten an Chancen und Risiken des Kapitalmarkts teil. In jüngster Zeit hat sich gezeigt, daß die neue Form der Alterssicherung keineswegs nur Zuwächse beschert: So mußten alle gewählten Anlageformen, also nicht

131 LIP Kap. $8, \S 1$.

132 Ebda., $\S \S 2$ und 3.

133 Ebda., §§ 4 ff.

134 Ebda., § 2.

135 LIP Kap. 4, § 7, und die Einzelheiten in Kap. 15, § 8. 
nur die eher riskanten Fonds, im Lauf der letzten Jahre ganz erhebliche Verluste verzeichnen. 2003 gelang erstmals 12,7 \% (ca. 633.000) der Sparer ein Anlagegewinn. Am 31.12.2003 gab es aber auch Rentenkonten mit mehr als $40 \%$ Verlust (ca. 55.000), die weitaus größte Gruppe bildeten mit über 2 Mio. die Konten mit einem Minus zwischen 10 und $20 \% 136$. Im Januar 2005 hatten nur $29 \%$ aller PPM-Sparer ein Plus auf ihrem Konto. Die Konten beliefen sich im Durchschnitt auf 4.300 SEK ${ }^{137}$. Im ersten Halbjahr 2008 verloren alle im Prämienrentensystem angesparten Fondsvermögen $16 \%$ an Wert 138 .

Das Wahlmodell der beitragsbestimmten Alterspension der Staatsbediensteten wurde im Nachgang zur Reform des allgemeinen Systems in Anlehnung an die Prämienrente eingeführt. Das bis 2003 geltende Dienstpensionenabkommen PA 91 sah nur eine leistungsbestimmte Alterspension vor, deren Höhe durch $10 \%$ der Gehälter bis zum 7,5fachen Grundbetrag, 65 \% für Gehälter bis zum 20-fachen Grundbetrag und 32,5 \% für Gehälter darüber bis zum 30-fachen Grundbetrag bestimmt wurde. Die neue Form der abgabenbestimmten Pension wurde also mit Kürzungen bei der leistungsbestimmten Rentenformel kompensiert.

\section{Bewertung}

Vorab ist zu konstatieren, daß in keinem der drei Nordischen Länder heute die Alterssicherung allein durch universalistische Systeme gewährleistet wird. Überall finden sich beitragsbezogene Zusatzsysteme. In allen Ländern haben sich daneben zumindest Rudimente der traditionellen Sondersysteme für Beamte erhalten. Diese sind grundsätzlich nicht durch Arbeitsvertrag, sondern z.T. nach verfassungsrechtlichen Vorgaben gesetzlich geregelt. In den Gesetzen finden sich dienstbedingte besondere Pflichten, etwa feste Probezeiten bei der Einstellung oder Disziplinarordnungen, aber auch Sonderregeln zur Beendigung der Dienstverhältnisse. Die jeweiligen Beamtengesetze regeln mit Ausnahme Schwedens, wo sie Gegenstand eines Kollektivvertrags ist, auch die Alterssicherung. Dabei ist für die Höhe der Pensionen stets das letzte erzielte Gehalt maßgebend. In allen Ländern liegt die Alterssicherung der Beamten über dem Niveau der allgemeinen Alterssicherung. Obgleich es (Ausnahme: Schweden) Beiträge der Bediensteten zur Finanzierung gibt, obliegt diese weit überwiegend dem Dienstherrn. Die Alterssicherungssysteme aller Länder waren in den letzten Jahren Gegenstand diverser Reformen. Dabei war nur die Reform in Schweden eine vollständige Neuordnung. Die Sondersysteme der Beamten waren davon aber durchweg nur moderat betroffen.

136 Zahlen nach PPM-Internetinformation „Årsstatistik 2003“ (zu entnehmen: http://www.ppm.nu).

$137^{\circ}$ PPM-Internetinformation „Årsstatistik 2006“.

138 S. Bjerre, Tungt fall för ppm-fonderna, Dagens Nyheter vom 8.Juli 2008, Ekonomi, S. 3. 


\title{
Die Altersversorgung von Beamten in der Russischen Föderation
}

\author{
Olga Chesalina
}

I. Grundlegung

1. System des Staatsdienstes in der Russischen Föderation

2. Bestehen eines „Berufsbeamtentums“ und Grundsätze der Beamtenverhältnisse

a) Historische Entwicklung des russischen Staatsdienstes

b) Verfassungsrechtliche und einfachgesetzliche Regelungen für die hoheitliche Aufgaben wahrnehmenden Staatsbediensteten

c) Verfassungsrechtliche und einfachgesetzliche Grundsätze des Staatsdienstes

3. Status der zivilen Staatsbediensteten und Kommunalbediensteten

a) Status der zivilen Staatsbediensteten

b) Status der Kommunalbediensteten

c) Verfassungsrechtliche Zulässigkeit der Statusregelungen

4. Erfordernis einer besonderen sozialen Sicherung der Beamten im Alter aufgrund der Besonderheiten der Stellung der Staatsbediensteten

II. Ausgestaltung der Altersabsicherung von Staatsbediensteten

1. Grundlegende Unterschiede zwischen den Alterssicherungssystemen für zivile Staatsbedienstete und Kommunalbedienstete und dem allgemeinen Alterssicherungssystem für Arbeitnehmer

a) Allgemeines System der Alterssicherung für Arbeitnehmer

b) Alterssicherung der zivilen Staatsbediensteten

c) Staatsbedienstete der Subjekte der Föderation und Kommunalbedienstete

2. Voraussetzungen des Erhalts einer Alterspension für zivile Staatsbedienstete

3. Leistungsbemessung der Alterspension für zivile Staatsbedienstete

4. Anpassungen der Alterspension für zivile Staatsbedienstete

5. Finanzierung der Alterssicherung der Staatsbediensteten

III. Auswertung 